\title{
Theory of Density Fluctuations in Strongly Radiative Plasmas
}

\author{
J. E. Cross,${ }^{1, *}$ P. Mabey, ${ }^{1}$ D. O. Gericke, ${ }^{2}$ and G. Gregori ${ }^{1, \dagger}$ \\ ${ }^{1}$ Clarendon Laboratory, University of Oxford, Parks Road, Oxford OX1 3PU, United Kingdom \\ ${ }^{2}$ Centre for Fusion, Space and Astrophysics, Department of Physics, \\ University of Warwick, Coventry CV4 7AL, United Kingdom
}

(Dated: February 7, 2016)

\begin{abstract}
Derivation of the dynamic structure factor, an important parameter linking experimental and theoretical work in dense plasmas, is possible starting from hydrodynamic equations. Here we obtain, by modifying the governing hydrodynamic equations, a new form of the dynamic structure factor which includes radiative terms. The inclusion of such terms has an effect on the structure factor at high temperatures, which suggests that its effect must be taken into consideration in such regimes.
\end{abstract}

Keywords: hydrodynamics - MHD - radiative transfer - structure factor

\section{INTRODUCTION}

Strongly radiative environments can be found in both laboratory and astrophysical systems. For example, in the laboratory, radiation transport must be considered in hohlraums [1] and more generally in inertial confinement fusion (ICF) experiments [2], as well as in tokamaks [3]. In astrophysics, radiation plays an important role in jets from young stellar objects [4], molecular clouds in star forming regions [5], and in plasmas surrounding active galactic nuclei, just to cite a few examples. In all of these cases, it is reasonable to expect that radiation changes the spectrum of the density fluctuations in some nontrivial manner, and thus neglecting radiation effects when diagnosing laboratory plasmas could significantly distort the inferred properties.

To illustrate this effect, we consider the special case of a dense plasma in thermodynamic equilibrium - the warm dense matter (WDM) regime - characterised by strongly correlated ions, and degenerate electrons [6]. These plasmas are found in the core of Jovian planets [7], in white dwarves, and in the crust of neutron stars [8]. Due to the high density, the radiation mean free path is small and the system is optically thick. Simulation and modelling of the dynamical behavior of WDM states, however, remains difficult, though there have been advances stemming from the availability of accurate laboratory data using high intensity lasers and pulsed power facilities [911], as well as newer computational approaches that allow density functional theory techniques to directly simulate the dynamics of thermal density fluctuations [12, 13], albeit a full inclusion of radiation processes in beyond current computational capabilities. In view of these limitations, a simplified theoretical model of density fluctuation in equilibrium with the radiation field could provide an important tool to benchmark computation models and to help the interpretation of experimental data.

\footnotetext{
*Electronic address: j.e.cross@physics.ox.ac.uk

${ }^{\dagger}$ Electronic address: gianluca.gregori@physics.ox.ac.uk
}

The fundamental thermodynamic quantity that describes the space and time dependent behavior of WDM, and other dense plasmas, is the dynamic structure factor (DSF), which is also proportional to the X-ray scattering cross section [15], and thus can be measured experimentally. X-ray Thomson scattering is an important diagnostic in many plasma physics experiments, both in the WDM regime and beyond (see [15] and references within). The comparison between the experimental and calculated DSF provides an important tool for the validation of theoretical models, toward a comprehensive understanding of these systems $[16,17]$. The DSF, $S(\boldsymbol{k}, \omega)$, is formally defined as [6]:

$$
S(\boldsymbol{k}, \omega)=\frac{1}{2 \pi N} \int e^{i \omega t}\langle\rho(\boldsymbol{k}, t) \rho(-\boldsymbol{k}, 0)\rangle d t
$$

which characterises thermal density fluctuations $\rho(\boldsymbol{k}, \omega)$ of wavevector $\boldsymbol{k}$, frequency $\omega$, where $N$ is the total number of particles, and $\langle\cdots\rangle$ represents an ensemble (or thermal) average.

An important case of density fluctuations is the one that corresponds to hydrodynamic fluctuations. By hydrodynamic we mean the regime where the spatial and temporal scales of the fluctuations are much larger than the mean particle separation and the mean time between collisions, respectively. This implies $k \rightarrow 0, \omega \rightarrow 0$. However, in practice, for hydrodynamics to be applicable, both the wavenumber and the frequency do not need to be negligibly small as long as the detailed kinetic behavior of the system can be ignored [18]. The hydrodynamic description of density fluctuations is important because it can be solved analytically [19, 20]. Moreover, the analytical results for the DSF in a dense viscous plasma are in very good agreement with molecular dynamics simulations [21]. On the other hand, there are several astrophysical and laboratory conditions where viscous hydrodynamics (i.e., the Navier-Stokes equations) may not be sufficient to capture all the relevant physical processes. These include, for example, the presence of dynamically strong magnetic fields, radiation transport as well as quantum effects $[22,23]$. 
In this paper we consider the equations of radiative hydrodynamics for matter in equilibrium with the radiation field [22] and analytically derive a modified form of the DSF for both optically thick and optically thin plasmas. We also assume, for the optically thick case, that the thermally-generated radiation field is described in the diffusive limit. We will show that, under certain conditions, radiation transport can induce significant changes in the dynamical response of the medium. As we have discussed above, this is particularly important since numerical calculations (either based on molecular dynamics or density functional theory) of the DSF in strongly radiative plasmas are not yet possible, due to the very demanding computational efforts. This paper begins with the general form of radiation hydrodynamic equations for an optically thick fluid in Section 2. In Section 3 we then derive the DSF by considering small fluctations in the plasma properties using a linearisation procedure. In Section 4 we examine an optically thin fluid. In Section 5 we discuss the specific conditions to when radiative effects ought to be considered in the DSF. We summarise our work in Section 6.

\section{GENERAL EQUATIONS FOR AN OPTICALLY THICK FLUID}

The equations for the conservation of mass, momentum and energy, as discussed in [22], but considering only viscous and radiative terms for an optically thick fluid (that is, a fluid where the mean free path of radiation is less than the spatial extent of the plasma) are given, respectively, by:

$$
\begin{gathered}
\frac{\partial \rho}{\partial t}+\nabla \cdot \rho \boldsymbol{u}=0 \\
\rho\left(\frac{\partial \boldsymbol{u}}{\partial t}+\boldsymbol{u} \cdot \nabla \boldsymbol{u}\right)=-\nabla p+\nabla \cdot \boldsymbol{\sigma}_{\nu}+f_{r a d}, \\
\rho\left(\frac{\partial \epsilon}{\partial t}+\boldsymbol{u} \cdot \nabla \epsilon\right)+\frac{\partial E_{R}}{\partial t}=-p \nabla \cdot \boldsymbol{u}-f_{r a d} \cdot \boldsymbol{u} \\
-\nabla \cdot\left[\boldsymbol{F}_{R}+\left(p_{R}+E_{R}\right) \cdot \boldsymbol{u}\right]+\nabla \cdot \boldsymbol{\sigma}_{\nu} \cdot \boldsymbol{u}-\nabla \cdot \boldsymbol{q}
\end{gathered}
$$

where $\rho$ is the mass density, $t$ is time, $\boldsymbol{u}$ the fluid velocity, $p$ the pressure, $\boldsymbol{\sigma}_{\nu}$ the stress tensor, $f_{\text {rad }}$ the radiation force on matter, $\epsilon$ the internal energy, $E_{R}$ the energy density of the radiation field, $\boldsymbol{F}_{R}$ the radiative energy flux, $p_{R}$ the radiation pressure, and $\boldsymbol{q}$ is the heat flux. (The radiation pressure is included for completeness, but, as we will see later, it does not have a large impact on the DSF in our example.) If radiation transport is considered in the diffusive limit, the above quantities can be written as:

$$
-\nabla p_{R}=f_{\text {rad }} ; \quad \boldsymbol{F}_{R}=-\frac{16 \sigma T^{3}}{3 \kappa_{R} \rho} \nabla T
$$

$$
p_{R}=\frac{E_{R}}{3}=\frac{4 \sigma T^{4}}{3 c},
$$

where $\sigma$ is the Stefan Boltzmann constant, $T$ the temperature, $c$ is the adiabatic sound speed, $\kappa_{R}$ is the Rosseland opacity, given the form [22]

$$
\kappa_{R}=\widetilde{\kappa_{R}} \rho^{a} T^{b}
$$

, where $\widetilde{\kappa_{R}}$ is a constant that depends on the material. In this case we consider the thermally generated radiation field of the plasma itself, rather than the action of an externally applied field. Substituting these relations into eqs. (2b) and (2c), after some simplification (see Appendix for details), we obtain:

$$
\begin{array}{r}
\rho\left(\frac{\partial \boldsymbol{u}}{\partial t}+\boldsymbol{u} \cdot \nabla \boldsymbol{u}\right)=-\left(\frac{c^{2}}{\gamma} \nabla \cdot \rho+\frac{c^{2}}{\gamma} \alpha_{T} \rho \nabla T\right) \\
+\eta_{1}\left[\nabla^{2} \boldsymbol{u}+\nabla(\nabla \cdot \boldsymbol{u})\right]+\eta_{2} \nabla(\nabla \cdot \boldsymbol{u})-\frac{4 \sigma}{3 c} \nabla T^{4}, \\
\rho C_{V}\left(\frac{D T}{D t}+\frac{\gamma-1}{\alpha_{T}} \nabla \cdot \boldsymbol{u}\right)+\frac{\partial}{\partial t}\left(\frac{4 \sigma T^{4}}{c}\right) \\
=-\nabla \cdot\left(\frac{16 \sigma T^{4}}{3 c} \boldsymbol{u}\right)+\nabla \cdot\left(\frac{16 \sigma T^{3}}{3 \kappa_{R} \rho} \nabla T\right) \\
-\boldsymbol{u} \nabla \cdot\left(\frac{4 \sigma T^{4}}{c}\right)+\nabla \cdot \boldsymbol{\sigma}_{\nu} \cdot \boldsymbol{u}+\kappa \nabla^{2} T
\end{array}
$$

where $\gamma$ is the adiabatic index, $\alpha_{T}$ the coefficient of thermal expansion, $\eta_{1,2}$ are the first and second coefficients of dynamic viscosity, $C_{V}$ is the heat capacity at constant volume, and $\kappa$ is the thermal conductivity. We use $D / D t$ as the material derivative, defined as $D / D t=(\partial / \partial t+\boldsymbol{u} \cdot \nabla)$. These are the general equations for an optically thick plasma, with terms relating to the radiative effects of the thermally self-generated radiation field, viscous nature of the fluid and convective heat transfer.

\section{FLUCTUATIONS}

We now consider small fluctuations, moving the system away from the equilibrium state, and linearise the above equations. We assume the system is at equilibrium at a density $\rho_{0}$, temperature $T_{0}$ and velocity $\boldsymbol{u}_{0}=0$ and small perturbations away from the equilibrium are added via:

$$
\delta \rho=\rho-\rho_{0}, \quad \delta T=T-T_{0}, \quad \delta \boldsymbol{u}=\boldsymbol{u},
$$

which then allows us to rewrite eq. (2a) as:

$$
\frac{\partial \rho_{0}}{\partial t}+\frac{\partial \delta \rho}{\partial t}+\nabla \cdot\left(\rho_{0} \boldsymbol{u}\right)+\nabla \cdot(\delta \rho \delta \boldsymbol{u})=0 .
$$

As we consider the fluctuations to be small we disregard fluctuation terms of order greater than one. Thus,

$$
\frac{\partial \delta \rho}{\partial t}+\nabla \cdot\left(\rho_{0} \boldsymbol{u}\right)=0
$$


Similarly we can write the momentum and energy equations respectively as (see Appendix for further details):

$$
\begin{aligned}
& \rho_{0} \frac{\partial \boldsymbol{u}}{\partial t}=-\frac{c_{0}^{2}}{\gamma_{0}}\left(\nabla \delta \rho+\alpha_{T 0} \rho_{0} \nabla \delta T\right) \\
& +\eta_{1_{0}}\left[\nabla^{2} \boldsymbol{u}+\nabla(\nabla \cdot \boldsymbol{u})\right]+\eta_{2_{0}} \nabla(\nabla \cdot \boldsymbol{u}) \\
& \quad-\frac{16 \sigma T_{0}^{3}}{3 c_{0}} \nabla \cdot \delta T, \quad(6 \mathrm{~b}) \\
& {\left[\left(\frac{16 \sigma T_{0}^{3}}{c_{0} \rho_{0} C_{V 0}}+1\right) \frac{\partial}{\partial t}-\left(\gamma_{0} \frac{\kappa_{0}}{\rho_{0} c_{V 0}}+\frac{16 \sigma T_{0}^{3}}{3 \kappa_{R 0} C_{V 0} \rho_{0}^{2}}\right) \nabla^{2}\right] \delta T} \\
& =-\nabla \cdot u\left(\frac{\gamma_{0}-1}{\alpha_{T 0}}+\frac{16 \sigma T_{0}^{4}}{3 c_{0} \rho_{0} C_{V 0}}\right) . \quad(6 \mathrm{c})
\end{aligned}
$$

In the above equations terms with the subscript " 0 " refers to quantities for equilibrium conditions.

To solve eqs. (6a) to (6c) we take the space (Fourier) and time (Laplace) transform,

$$
\widetilde{\delta x}_{k}(s)=\int_{0}^{\infty} \mathrm{d} t e^{-s t} \int_{-\infty}^{+\infty} \mathrm{d}^{3} r e^{i \boldsymbol{k} \cdot \boldsymbol{r}} \delta x(\boldsymbol{r}, t)
$$

where $x$ is a chosen plasma property (either density, temperature or velocity), with the tilde indicating the transformed property, and $s=\varepsilon+i \omega$ is the complex Laplace variable. Following the method discussed in Boon and Yip (1991) [24], we define our independent variables as $\delta \rho, \boldsymbol{j}^{\boldsymbol{M}}=\rho_{0} \boldsymbol{u}$ and $g=\rho_{0} \delta T$. This gives us the following equations:

$$
\begin{gathered}
s \widetilde{\delta \rho_{k}}(s)+i \boldsymbol{k} \cdot \boldsymbol{j}^{\boldsymbol{M}}(s)=\delta \rho_{k}(0), \\
\left(s+\nu_{l_{0}} k^{2}\right) \tilde{\boldsymbol{j}}_{\boldsymbol{k}}^{\boldsymbol{M}}(s)+\frac{c_{0}^{2}}{\gamma_{0}} i \boldsymbol{k} \widetilde{\delta \rho_{k}}(s) \\
+i \boldsymbol{k}\left(\frac{c_{0}^{2} \alpha_{T 0}}{\gamma_{0}}+\frac{16 \sigma T_{0}^{3}}{3 c_{0} \rho_{0}}\right) \tilde{g}_{k}(s)=\boldsymbol{j}_{\boldsymbol{k}}^{M}(0), \quad(7 \mathrm{~b}) \\
{\left[\left(\frac{16 \sigma T_{0}^{3}}{c_{0} \rho_{0} C_{V 0}}+1\right) s+k^{2}\left(\gamma_{0} \chi_{0}+\frac{16 \sigma T_{0}^{3}}{3 \kappa_{R} \rho_{0}^{2} C_{V 0}}\right)\right] \tilde{g}_{k}(s)} \\
+i \boldsymbol{k}\left(\frac{\gamma_{0}-1}{\alpha_{T 0}}+\frac{16 \sigma T_{0}^{4}}{3 c_{0} \rho_{0} C_{V 0}}\right) \cdot \boldsymbol{j}_{\boldsymbol{k}}^{\boldsymbol{M}}(s) \\
=\left(\frac{16 \sigma T_{0}^{3}}{c_{0} \rho_{0} C_{V 0}}+1\right) g_{k}(0), \quad(7 \mathrm{c})
\end{gathered}
$$

where $\nu_{l_{0}}=\left(2 \eta_{1_{0}}+\eta_{2_{0}}\right) / \rho_{0}$ is the longitudinal viscosity, and $\chi_{0}=\kappa_{0} / \rho_{0} C_{P 0}$ is the thermal diffusivity $\left(C_{P 0}\right.$ is the heat capacity at constant pressure). These equations can be solved for $\widetilde{\delta} \rho_{k}(s)$ (see the Appendix for details). Since terms involving cross-correlation of $\delta \rho$ and $\boldsymbol{j}^{\boldsymbol{M}}$, or $g$, vanish, as they are all independent variables, the density-density correlation function is given by:

$$
\frac{\left\langle\delta \rho_{k}^{*}(0) \tilde{\delta} \rho_{k}(s)\right\rangle}{\left\langle\delta \rho_{k}^{*}(0) \delta \rho_{k}(0)\right\rangle}=\frac{\left(s+\nu_{l_{0}} k^{2}\right)\left(\Delta s+X_{T h} k^{2}\right)+k^{2}\left(\frac{c_{0}^{2} \alpha_{T 0}}{\gamma_{0}}+16 T_{T h}\right)\left(\frac{\gamma_{0}-1}{\alpha_{T 0}}+\frac{16 T_{T h} T_{0}}{C_{V 0}}\right)}{s\left[\left(s+\nu_{l_{0}} k^{2}\right)\left(\Delta s+X_{T h} k^{2}\right)+k^{2}\left(\frac{c_{0}^{2} \alpha_{T 0}}{\gamma_{0}}+16 T_{T h}\right)\left(\frac{\gamma_{0}-1}{\alpha_{T 0}}+\frac{16 T_{T h} T_{0}}{C_{V 0}}\right)\right]+\frac{\left(c_{0} k\right)^{2}}{\gamma_{0}}\left(\Delta s+X_{T h} k^{2}\right)}
$$

Where we have defined:

$$
\begin{gathered}
\Delta=1+\frac{16 \sigma T_{0}^{3}}{c_{0} \rho_{0} C_{V 0}} \\
X_{T h}=\gamma_{0} \chi_{0}+\frac{16 \sigma T_{0}^{3}}{3 \kappa_{R 0} \rho_{0}^{2} C_{V 0}} \\
T_{T h}=\frac{\sigma T_{0}^{3}}{3 c_{0} \rho_{0}}
\end{gathered}
$$

Defining $S(k)=\left\langle\delta \rho_{k}^{*}(0) \delta \rho_{k}(0)\right\rangle$ (the static structure factor), the dynamic structure factor is then obtained by taking the limit

$$
\frac{S(k, \omega)}{S(k)}=2 \Re\left[\lim _{\varepsilon \rightarrow 0} \frac{\left\langle\delta \rho_{k}^{*}(0) \widetilde{\delta \rho_{k}}(s=\varepsilon+i \omega)\right\rangle}{\left\langle\delta \rho_{k}^{*}(0) \delta \rho_{k}(0)\right\rangle}\right] .
$$

This can be separated into partial fractions, and in the limit of small $k$, becomes

$$
\begin{array}{r}
\frac{S(k, \omega)}{S(k)}=\left[1-\frac{1}{\gamma_{0}}\left(\frac{\Delta c_{0} k}{C_{Q}}\right)^{2}\right] \frac{2 \xi_{Q} X_{T h} k^{2}}{\left(X_{T h} k^{2}\right)^{2}+\omega^{2}} \\
+\frac{1}{\gamma_{0}}\left(\frac{\Delta c_{0} k}{C_{Q}}\right)^{2}\left\{\frac{\Gamma_{R} k^{2}}{\left(\Gamma_{R} k^{2}\right)^{2}+\left[\omega+\left(\frac{C_{Q}}{\sqrt{\Delta}}\right)\right]^{2}}\right. \\
\left.+\frac{\Gamma_{R} k^{2}}{\left(\Gamma_{R} k^{2}\right)^{2}+\left[\omega-\left(\frac{C_{Q}}{\sqrt{\Delta}}\right)\right]^{2}}\right\},
\end{array}
$$


where we have defined:

$$
\begin{gathered}
C_{Q}^{2}=\frac{c_{0}^{2} k^{2}}{\gamma_{0}}\left[\left(\gamma_{0}-1\right)+\Delta+\frac{16 T_{T h} T_{0} \alpha_{T 0}}{C_{V 0}}\right] \\
+k^{2}\left[\frac{16 T_{T h}\left(\gamma_{0}-1\right)}{\alpha_{T 0}}+\frac{256 T_{T h}^{2} T_{0}}{C_{V 0}}\right] ; \\
\xi_{Q}=\frac{c_{0}^{2} k^{2}}{\gamma_{0}} \frac{1}{C_{Q}^{2}} \\
\Gamma_{R}=\frac{X_{T h}\left(\frac{1}{\Delta}-\xi_{Q}\right)+\nu_{\ell_{0}}}{2} .
\end{gathered}
$$

As we will discuss below, this representation of the structure factor has a similar form as the one derived for the case of pure hydrodynamic fluctuations.

\section{GENERAL EQUATIONS FOR AN OPTICALLY THIN FLUID}

Optically thin conditions can be found for tenuous plasmas found in laboratory and astrophysical systems. For the sake of generality, we will now derive the the density fluctuation spectrum for an optically thin plasma, but identify later that changes induced in the DSF are minimal for this condition. Following the same approach as before, we now focus on the case of a fluid where the mean free path of radiation is far greater than the spatial extent of the plasma [22]. The relevant equations are:

$$
\begin{gathered}
\frac{\partial \rho}{\partial t}+\nabla \cdot \rho \boldsymbol{u}=0 \\
\rho\left(\frac{\partial \boldsymbol{u}}{\partial t}+\boldsymbol{u} \cdot \nabla \boldsymbol{u}\right)=-\nabla p+\nabla \cdot \boldsymbol{\sigma}_{\nu}, \\
\rho\left(\frac{\partial \epsilon}{\partial t}+\boldsymbol{u} \cdot \nabla \epsilon\right)=-p \nabla \cdot \boldsymbol{u}+\nabla \cdot \boldsymbol{\sigma}_{\nu} \cdot \boldsymbol{u}+\kappa \nabla^{2} T-\ell_{\Lambda},
\end{gathered}
$$

where we assume that radiative losses are treated in terms of a cooling function, $\ell_{\Lambda}$, which is parametrized as

$$
\ell_{\Lambda}=\rho \kappa_{P} \sigma T^{4}
$$

where $\kappa_{P}$ is the Planck mean opacity, which we assume to have the form $\kappa_{P}=\widetilde{\kappa_{P}} \rho^{a} T^{b}[22]$, with $\widetilde{\kappa_{P}}$ a material dependent constant. It is thus obvious that, for optically thin plasmas, the radiation only acts as a loss term in the energy, but has no significant impact on the momentum of the plasma.

We now proceed by linearising the above equations, and take small fluctuation of the equilibrium state, as for the optically thick case, $\delta \rho=\rho-\rho_{0}, \delta T=T-T_{0}, \delta \boldsymbol{u}=\boldsymbol{u}$, which gives

$$
\frac{\partial \delta \rho}{\partial t}+\nabla \cdot\left(\rho_{0} \boldsymbol{u}\right)=0
$$

$$
\begin{aligned}
\rho_{0} \frac{\partial \boldsymbol{u}}{\partial t} & =-\frac{c_{0}^{2}}{\gamma_{0}}\left(\nabla \cdot \delta \rho+\alpha_{T 0} \rho_{0} \nabla \delta T\right) \\
& +\eta_{1_{0}}\left[\nabla^{2} \boldsymbol{u}+\nabla(\nabla \cdot \boldsymbol{u})\right]+\eta_{2_{0}} \nabla(\nabla \cdot \boldsymbol{u})
\end{aligned}
$$

$$
\begin{array}{r}
{\left[\frac{\partial}{\partial t}-\frac{\kappa_{0}}{C_{V 0} \rho_{0}} \nabla^{2}+(b+4) \frac{\kappa_{P 0} \rho_{0}}{C_{V 0}} \sigma T_{0}^{3}\right] \delta T+\frac{\gamma_{0}-1}{\alpha_{T 0}} \nabla \cdot \boldsymbol{u}} \\
=-\frac{\kappa_{P 0} \sigma}{\rho_{0} C_{V 0}}\left[\rho_{0} T_{0}^{4}+(a+1) T_{0}^{4} \delta \rho\right], \quad(14 \mathrm{c})
\end{array}
$$

Taking again our independent variables to be $\delta \rho, \boldsymbol{j}^{\boldsymbol{M}}=$ $\rho_{0} \boldsymbol{u}$ and $g=\rho_{0} \delta T$, and applying Laplace and Fourier transforms to eq. (14) gives

$$
s \widetilde{\delta} \rho_{k}(s)+i \boldsymbol{k} \cdot \boldsymbol{j}^{\boldsymbol{M}}(s)=\delta \rho_{k}(0)
$$

$$
\left(s+\nu_{l_{0}} k^{2}\right) \tilde{\boldsymbol{j}}_{\boldsymbol{k}}^{\boldsymbol{M}}(s)+\frac{c_{0}^{2}}{\gamma_{0}} i \boldsymbol{k}\left[\widetilde{\delta \rho_{k}}(s)+\alpha_{T 0} \tilde{g}_{k}(s)\right]=\boldsymbol{j}_{\boldsymbol{k}}^{\boldsymbol{M}}(0),
$$

$$
\begin{aligned}
{\left[s+\gamma_{0} \chi_{0} k^{2}\right.} & \left.+(b+4) \frac{\kappa_{P 0}}{C_{V 0}} \sigma T_{0}^{3}\right] \tilde{g}_{k}(s)+\frac{\gamma_{0}-1}{\alpha_{T 0}} i \boldsymbol{k} \cdot \boldsymbol{j}_{\boldsymbol{k}}^{M}(s) \\
& +\frac{\kappa_{P 0}}{C_{V 0}}(a+1) \sigma T_{0}^{4} \delta \tilde{\rho}_{k}(s)=g_{k}(0) . \quad(15 \mathrm{c})
\end{aligned}
$$

As for the optically thick case, we can solve these equations to get $\widetilde{\delta} \rho_{k}(s)$ (for more detail on the procedure see Appendix) and then construct the normalized correlation function: 


$$
\begin{aligned}
& \frac{\left\langle\delta \rho_{k}^{*}(0) \widetilde{\delta} \rho_{k}(s)\right\rangle}{\left\langle\delta \rho_{k}^{*}(0) \delta \rho_{k}(0)\right\rangle} \\
= & \frac{\left(s+\nu_{l_{0}} k^{2}\right)\left[s+\gamma_{0} \chi_{0} k^{2}+(b+4) \frac{\kappa_{P 0}}{C_{V 0}} \sigma T_{0}^{3}\right]+\frac{\gamma_{0}-1}{\gamma_{0}} c_{0}^{2} k^{2}}{s\left[\left(s+\nu_{l_{0}} k^{2}\right)\left(s+\gamma_{0} \chi_{0} k^{2}+(b+4) \frac{\kappa_{P 0}}{C_{V 0}} \sigma T_{0}^{3}\right)+\frac{\gamma_{0}-1}{\gamma_{0}} c_{0}^{2} k^{2}\right]+\frac{c_{0}^{2} k^{2}}{\gamma_{0}}\left[s+\gamma_{0} \chi_{0} k^{2}+(b+4) \frac{\kappa_{P 0}}{C_{V 0}} \sigma T_{0}^{3}-\alpha_{T 0} \frac{\kappa_{P 0}}{C_{V 0}}(a+1) \sigma T_{0}^{4}\right]} .
\end{aligned}
$$

The corresponding dynamic structure factor is then obtained by taking the same limit as in eq. (10). Unlike in the optically thick and hydrodynamic cases, this form of the DSF cannot be separated into partial fractions.

\section{DISCUSSION}

It is helpful to compare our results to the pure hydrodynamic case. In the latter it is straightforward to show that $[24]$

$$
\begin{gathered}
\frac{\left\langle\delta \rho_{k}^{*}(0) \tilde{\delta} \rho_{k}(s)\right\rangle}{\left\langle\delta \rho_{k}^{*}(0) \delta \rho_{k}(0)\right\rangle}= \\
\frac{\left(s+\nu_{l_{0}} k^{2}\right)\left(s+\gamma_{0} \chi_{0} k^{2}\right)+\frac{\gamma_{0}-1}{\gamma_{0}} c_{0}^{2} k^{2}}{s\left[\left(s+\nu_{l_{0}} k^{2}\right)\left(s+\gamma_{0} \chi_{0} k^{2}\right)+\frac{\gamma_{0}-1}{\gamma_{0}} c_{0}^{2} k^{2}\right]+\frac{c_{0}^{2} k^{2}}{\gamma_{0}}\left(s+\gamma_{0} \chi_{0} k^{2}\right)} .
\end{gathered}
$$

By expanding in $k$ and discarding term of order higher than $k^{2}$, the above expression can be expanded in partial fractions. This leads to:

$$
\begin{aligned}
& \frac{S(k, \omega)}{S(k)}=\frac{\gamma_{0}-1}{\gamma_{0}} \frac{2 \chi_{0} k^{2}}{\omega^{2}+\left(\chi_{0} k^{2}\right)^{2}}+ \\
& \frac{1}{\gamma_{0}}\left[\frac{\Gamma k^{2}}{\left(\Gamma k^{2}\right)^{2}+\left(\omega+c_{0} k\right)^{2}}+\frac{\Gamma k^{2}}{\left(\Gamma k^{2}\right)^{2}+\left(\omega-c_{0} k\right)^{2}}\right],
\end{aligned}
$$

where

$$
\Gamma=\frac{\left(\gamma_{0}-1\right) \chi_{0}+\nu_{\ell_{0}}}{2}
$$

Comparing eq. (11) for an optically thick plasma, and eq. (18) for the hydrodynamic case without radiation, we indeed recover the same structure of the DSF. In the hydrodynamic case this corresponds to two Brillouin peaks, whose position is given by the dispersion relation and the width determined by the parameter $\Gamma$. There is also an elastic (entropy) peak, the strength of which is set by $\gamma_{0}$ and $\chi_{0}$. For an optically thick plasma, the same threepeak structure is present, but additional factors related to radiation transport are now affecting their position, width and intensity.

The radiative modification of the structure factor for a dense aluminum plasma is illustrated in Figure 1, where
TABLE I: List of plasma parameters used in generating figure 1. The thermal diffusivity, kinematic viscosity and opacity expressions are taken from [25], [26] and [27] respectively.

\begin{tabular}{lcc}
\hline Parameter & \multicolumn{2}{c}{ Value } \\
\hline Atomic Number & $\mathrm{A}$ & 13 \\
Mass Density & $\rho$ & $7.0 \mathrm{~g} / \mathrm{cc}$ \\
Temperature & $\mathrm{T}$ & $5-75 \mathrm{eV}$ \\
Plasma Frequency & $\omega_{p}$ & $0.15 \mathrm{fs}^{-1}$ \\
Wavenumber & $\boldsymbol{k}$ & $2.1 \times 10^{10} \mathrm{~m}^{-1}$ \\
Adiabatic Index & $\gamma$ & 1.79 \\
Coloumb Logarithm & $\Lambda$ & 10 \\
\hline Thermal Diffusivity $\left[\mathrm{m}^{2} / \mathrm{s}\right]$ & $\chi_{t h}$ & $3.3 \times 10^{-7} \frac{A T^{5 / 2}}{Z(Z+1) \rho \Lambda}$ \\
Kinematic Viscosity $\left[\mathrm{m}^{2} / \mathrm{s}\right]$ & $\nu_{\ell}$ & $3.3 \times 10^{-9} \frac{A^{1 / 2} T^{5 / 2}}{Z^{4} \rho \Lambda}$ \\
Planck Mean Opacity $\left[\mathrm{m}^{2} / \mathrm{kg}\right]$ & $\kappa_{P}$ & $6.01 \times 10^{7} \rho^{0.48} T^{-2.42}$ \\
Rosseland Mean Opacity $\left[\mathrm{m}^{2} / \mathrm{kg}\right]$ & $\kappa_{R}$ & $1.04 \times 10^{7} \rho^{0.48} T^{-2.48}$ \\
\hline
\end{tabular}

we have estimated the relevant transport coefficients as indicated in Table I. The ionisation state was taken from the PrOpacEos tables [28]. In Figure 1, the calculated DSF's have all been normalised to the pure hydrodynamic case. In each case the density is fixed at $7.0 \mathrm{~g} / \mathrm{cc}$, and the temperature is allowed to change. As expected, at low temperatures there is no effect on the shape of the structure factor. As we increase the temperature the ion-acoustic resonance becomes narrower. This effect can be related to the $T^{4}$ dependence of the radiative terms: being too small at low temperature to have a significant effect. Since the radiation acts as a heat bath, it reduces the thermal fluctuations of the plasma, and thus sharpens the peaks. The opacity acts as a coupling parameter, and so explains why this effect is more pronounced in the optically thick case. Assuming the same conditions, but for an optically thin plasma, produces a DSF that is essentially the same as the one we have calculated for ideal hydrodynamics.

Figure 2 also shows the effect of the radiation on the structure factor when moving to denser material. The conditions are equivalent to those in figure 1 , but the density is now varied between 0.1 and $10 \mathrm{~g} / \mathrm{cc}$ at a fixed temperature of $50 \mathrm{eV}$. Again the all curves are normalised to the hydrodynamic case. Similarly to the case of increasing temperature, we see an increased effect of radi- 


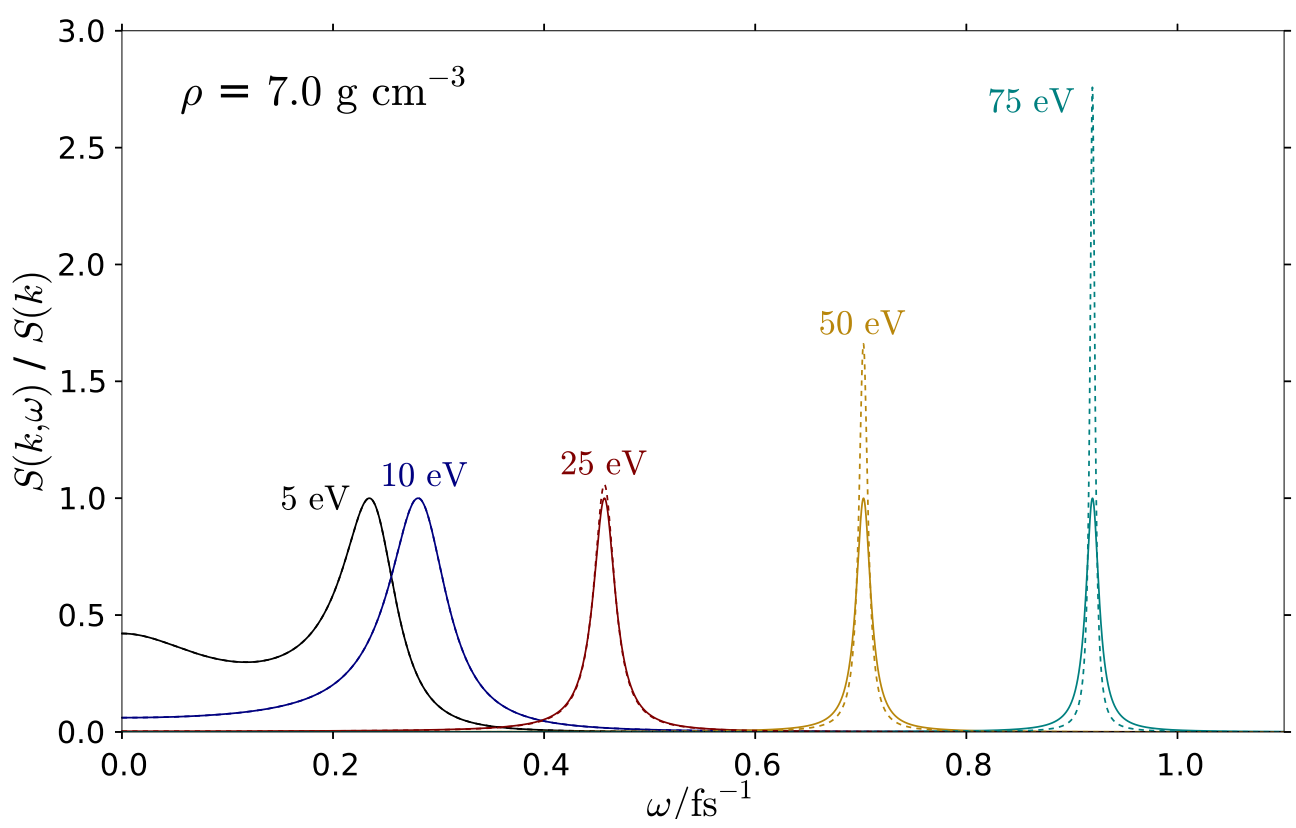

FIG. 1: Dynamic Structure factor versus frequency for a range of temperatures, for an aluminium plasma with density fixed at $7 \mathrm{~g} \mathrm{~cm}^{-3}$. The hydrodynamic case is shown with a solid line, while the (optically thick) radiative case is shown with a dashed line. Each line is scaled so that the hydrodynamic case has its maximum at 1.0. We notice that the height of the peaks increases and their widths narrows as the temperature becomes larger.

ation upon going to higher densities. The radiation acts to narrow the peaks, and increase the intensity. This is likely to be because of the increased density improving the coupling to the radiation field.

To determine under what conditions we would expect to see a noticeable effect of the radiation on the DSF, it is useful to estimate the relevant characteristic dimensionless numbers [22]. The Mihalas number, $R$, gives the ratio of the material (ram) pressure to the radiative pressure in the fluid. The optically thick number, $\Pi_{T h i c k}$, gives the ratio of the material enthalpy flux to the radiative energy flux. For the case of a $7.0 \mathrm{~g} \mathrm{~cm}^{-3}$ aluminium plasma at $50 \mathrm{eV}$, as seen in figure 1 , we estimate values of $R \sim 10^{4}$ and $\Pi_{\text {Thick }} \sim 10^{-10}$, using the thermal speed as the typical fluid velocity. This suggests that while the radiative pressure is not dominant, there is a significant effect from radiative flux loss, which dominates over the material flux. This explains the 'thinning' of the peaks in the DSF.

\section{CONCLUDING REMARKS}

In this paper we have derived, using the relevant radiation hydrodynamic equations, an analytical form of the dynamic structure factor in the presence of either an optically thin or thick fluid. Inclusion of radiation terms is shown to have noticeable effects in the optically thick case at higher temperatures. Under these conditions, the DSF is shown to have the same three-peak structure as in the case of pure hydrodynamic fluctuations. Radiation thus acts to modify the width and heights of the ion-acoustic resonance, and in particular, a narrowing of the resonances by cooling is observed in presence of strong radiation. For the optically thin case we have derived a general expression for the DSF, but further expansion in partial fractions is not possible. Moreover, in this regime, the changes induced by radiation play a modest effect. The results suggest that experimentally, when using X-Ray scattering as a diagnostic, radiative effects must included for hot and optically thick plasmas in order to accurately infer plasma properties, but can be neglected in optically thin plasmas.

\section{Acknowledgments}

The research leading to these results has received funding from the European Research Council under the European Community's Seventh Framework Programme (FP7/2007-2013) / ERC grant agreement no. 256973. Partial support from AWE plc is also acknowledged. 


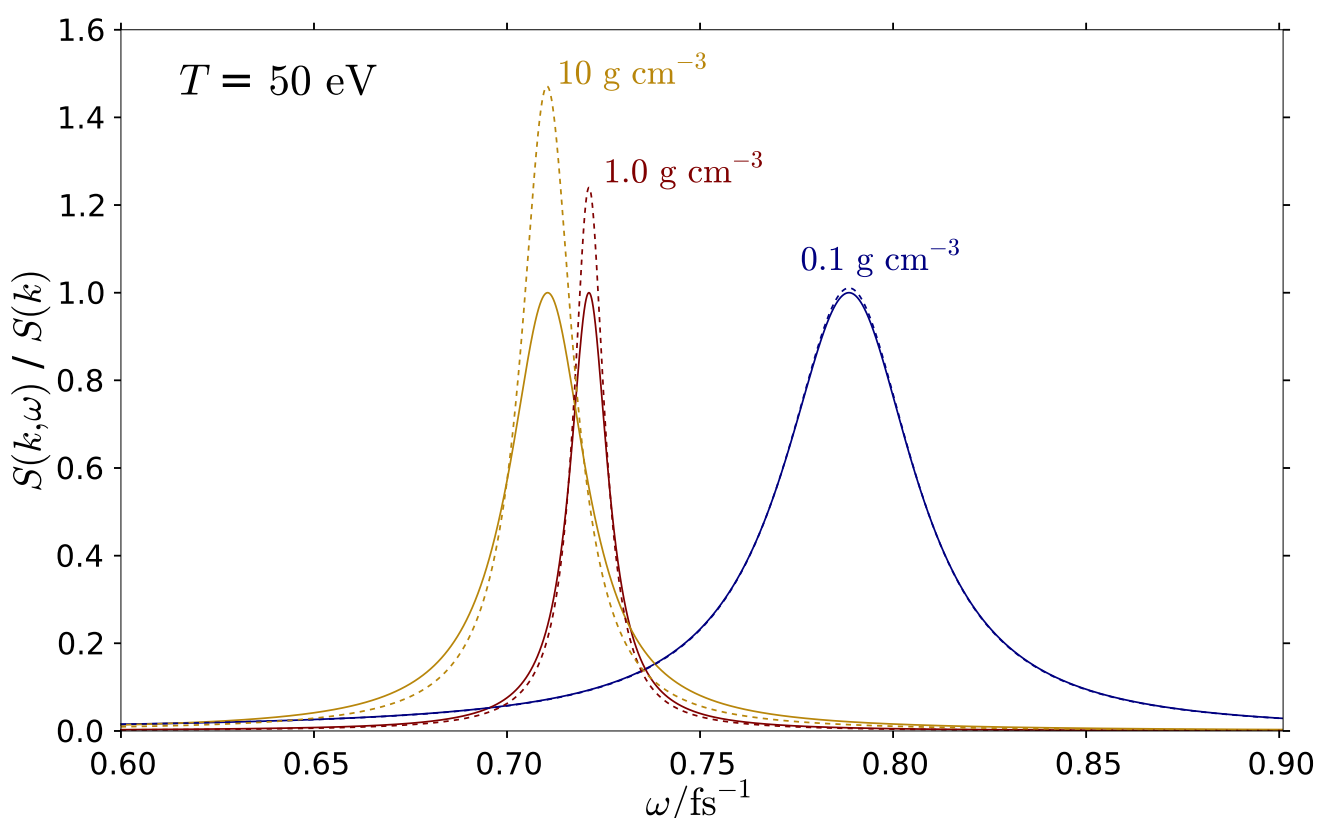

FIG. 2: Dynamic Structure factor versus frequency for a range of densities, for an aluminium plasma with temperature fixed at $50 \mathrm{eV}$. The hydrodynamic case is shown with a solid line, while the (optically thick) radiative case shown with a dashed line. Each line is scaled so that the hydrodynamic case has its maximum at 1.0. We notice that the height of the peaks increases and their widths narrows as the density becomes larger.

\section{Appendix A: Momentum Equation}

Here we outline the procedure for calculating the dynamic structure factor using, as an example, the radiation hydrodynamic equations in an optically thin fluid.

We start from rewriting eq. (13b) explicitly

$$
\rho\left(\frac{\partial \boldsymbol{u}}{\partial t}+\boldsymbol{u} \cdot \nabla \boldsymbol{u}\right)=-\nabla p+\eta_{1}\left[\nabla^{2} \boldsymbol{u}+\nabla(\nabla \cdot \boldsymbol{u})\right]+\eta_{2} \nabla(\nabla \cdot \boldsymbol{u}),
$$

where $\rho$ is the mass density, $t$ the time, $\boldsymbol{u}$ the velocity, $p$ the pressure and $\eta_{i}(i=1,2)$ are the first and second coefficients of viscosity, respectively. We then use the thermodynamic relations:

$$
\nabla p=\left(\frac{\partial p}{\partial \rho}\right)_{T} \nabla \rho+\left(\frac{\partial p}{\partial T}\right)_{\rho} \nabla T, \quad\left(\frac{\partial p}{\partial \rho}\right)_{T}=\frac{C_{V}}{C_{P}}\left(\frac{\partial p}{\partial \rho}\right)_{\mathcal{S}}=\frac{c^{2}}{\gamma}, \quad\left(\frac{\partial p}{\partial T}\right)_{\rho}=\left(\frac{\partial p}{\partial \rho}\right)_{T}\left(\frac{\partial \rho}{\partial T}\right)_{p}=\frac{c^{2}}{\gamma} \alpha_{T} \rho
$$

where $C_{V}$ is the heat capacity at constant volume, $C_{P}$ is the heat capacity at constant pressure, $\mathcal{S}$ is the entropy, $c$ is the adiabatic sound speed, $\gamma$ is the adiabatic index, and $\alpha_{T}$ is the coefficient of thermal expansion.

We now use these relations in equation A1 to give

$$
\rho\left(\frac{\partial \boldsymbol{u}}{\partial t}+\boldsymbol{u} \cdot \nabla \boldsymbol{u}\right)=-\left(\frac{c^{2}}{\gamma} \nabla \cdot \rho+\frac{c^{2}}{\gamma} \alpha_{T} \rho \nabla T\right)+\eta_{1}\left[\nabla^{2} \boldsymbol{u}+\nabla(\nabla \cdot \boldsymbol{u})\right]+\eta_{2} \nabla(\nabla \cdot \boldsymbol{u}),
$$

Linearising with $\delta \rho=\rho-\rho_{0}, \delta T=T-T_{0}, \delta u=u$, and only keeping terms to first order in the fluctuations, gives equation $14 \mathrm{~b}$. 


\section{Appendix B: Energy Equation}

Starting from equation $13 \mathrm{c}$ we use the thermodynamic relations

$$
d \epsilon=\left(\frac{\partial \epsilon}{\partial \rho}\right)_{\mathcal{S}} d \rho+\left(\frac{\partial \epsilon}{\partial \mathcal{S}}\right)_{\rho} d \mathcal{S}, \quad\left(\frac{\partial \epsilon}{\partial \rho}\right)_{\mathcal{S}}=\frac{p}{\rho^{2}}, \quad\left(\frac{\partial \epsilon}{\partial \mathcal{S}}\right)_{\rho}=T
$$

where $\epsilon$ is the internal energy per unit mass, and thus the continuity equation (13a) becomes

$$
\rho T \frac{d \mathcal{S}}{d t}=\nabla \cdot \boldsymbol{\sigma}_{\nu} \cdot \boldsymbol{u}-\nabla \cdot \boldsymbol{q}-\ell_{\Lambda}
$$

Next, we assume a specific form for the heat flux, $\boldsymbol{q}$, and then write the entropy in terms of $\rho$ and $T$, i.e.,

$$
\begin{gathered}
\nabla \cdot \boldsymbol{q}=-\kappa \nabla^{2} T, \quad d \mathcal{S}=\left(\frac{\partial \mathcal{S}}{\partial \rho}\right)_{T} d \rho+\left(\frac{\partial \mathcal{S}}{\partial T}\right)_{\rho} d T \\
\left(\frac{\partial \mathcal{S}}{\partial \rho}\right)_{T}=-\frac{1}{\rho^{2}}\left(\frac{\partial p}{\partial T}\right)_{\rho}, \quad\left(\frac{\partial \mathcal{S}}{\partial T}\right)_{\rho}=\frac{C_{V}}{T}, \quad\left(\frac{\partial p}{\partial T}\right)_{\rho}=\frac{C_{P}-C_{V}}{\alpha_{T} T} \rho
\end{gathered}
$$

where $\kappa$ is the thermal conductivity.

Substituting these into the above equation, and making use of the continuity equation again, gives:

$$
\frac{\partial T}{\partial t}+\boldsymbol{u} \cdot \nabla T=\frac{1}{C_{V} \rho}\left(\nabla \cdot \boldsymbol{\sigma}_{\nu} \cdot \boldsymbol{u}+\kappa \nabla^{2} T-\rho \kappa_{P} \sigma T^{4}\right)-\frac{\gamma-1}{\alpha_{T}} \nabla \cdot \boldsymbol{u} .
$$

Linearising with $\delta \rho=\rho-\rho_{0}, \delta T=T-T_{0}, \delta u=u$, and only keeping terms to first order in the fluctuations gives equation $14 \mathrm{c}$.

\section{Appendix C: Solving for Transformed Fluctuation Density, $\widetilde{\delta} \rho_{k}(s)$}

We can solve eqs. (15a) to (15c) for $\widetilde{\delta}_{k}(s)$. Using Cramer's Rule, we have

$$
\widetilde{\delta} \rho_{k}(s)=\frac{|N(k, s)|}{|M(k, s)|}
$$

where we define the matrices $N(k, s)$ and $M(k, s)$ from eqs. (15a) to (15c) as (with independent variables $\rho$, $\boldsymbol{j}^{\boldsymbol{M}}=\rho_{0} \boldsymbol{u}$ and $\left.g=\rho_{0} \delta T\right)$ :

$$
\begin{aligned}
& M(k, s)=\left[\begin{array}{ccc}
s & i \boldsymbol{k} & 0 \\
\frac{c_{0}^{2}}{\gamma_{0}} i \boldsymbol{k} & s+\nu_{l_{0}} k^{2} & \frac{\alpha_{T 0} c_{0}^{2}}{\gamma_{0}} i \boldsymbol{k} \\
\frac{\kappa_{P 0}}{C_{V 0}}(a+1) \sigma T_{0}^{4} & \frac{\gamma_{0}-1}{\alpha_{T 0}} i \boldsymbol{k} & s+\gamma_{0} \chi_{0} k^{2}+(4+b) \frac{\kappa_{P 0}}{C_{V 0}} \rho T_{0}^{3}
\end{array}\right] \\
& N(k, s)=\left[\begin{array}{ccc}
\delta \rho_{k}(0) & i \boldsymbol{k} & 0 \\
j_{\boldsymbol{k}}^{M}(0) & s+\nu_{l_{0}} k^{2} & \frac{\alpha_{T 0} c_{0}^{2}}{\gamma_{0}} i \boldsymbol{k} \\
g_{k}(0) & \frac{\gamma_{0}-1}{\alpha_{T 0}} i \boldsymbol{k} & s+\gamma_{0} \chi_{0} k^{2}+(4+b) \frac{\kappa_{P 0}}{C_{V 0}} \rho_{0} T_{0}^{3}
\end{array}\right]
\end{aligned}
$$

which gives,

$$
\widetilde{\delta} \rho_{k}(s)=\frac{\delta \rho_{k}(0)\left[\left(s+\nu_{l_{0}} k^{2}\right)\left(s+\gamma_{0} \chi_{0} k^{2}+(4+b) \frac{\kappa_{P 0}}{C_{V 0}} \sigma T_{0}^{3}\right)+\frac{\gamma_{0}-1}{\gamma_{0}} c_{0}^{2} k^{2}\right]-i \boldsymbol{k}\left[\boldsymbol{j}_{\boldsymbol{k}}^{M}(0)\left(s+\gamma_{0} \chi_{0} k^{2}+(4+b) \frac{\kappa_{P 0}}{C_{V 0}} \sigma T_{0}^{3}\right)-g_{k}(0) \frac{\alpha_{T 0} c_{0}^{2}}{\gamma_{0}} i \boldsymbol{k}\right.}{s\left[\left(s+\nu_{l_{0}} k^{2}\right)\left(s+\gamma_{0} \chi_{0} k^{2}+(4+b) \frac{4 \kappa_{P 0}}{C_{V 0}} \sigma T_{0}^{3}\right)+\frac{\gamma_{0}-1}{\gamma_{0}} c_{0}^{2} k^{2}\right]-i \boldsymbol{k}\left[\frac{c_{0}^{2}}{\gamma_{0}} i \boldsymbol{k}\left(s+\gamma_{0} \chi_{0} k^{2}+(4+\beta) \frac{\kappa_{P 0}}{C_{V 0}} \sigma T_{0}^{3}-\frac{\alpha_{T 0} \kappa_{P 0}}{C_{V 0}}(a+1) \sigma T_{0}^{4}\right)\right]}
$$


where $\nu_{\ell_{0}}$ is the kinematic viscosity, $\chi_{0}=\kappa_{0} / \rho_{0} C_{P 0}$ is the thermal diffusivity.

[1] Glenzer, S. H. et al. Phys. Rev. Lett., 106, 085004 (2011).

[2] Lindl, J. D., Amendt, P., Berger, R. L., Glendinning, S. G., Glenzer, S. H., Haan, S. W., Kauffman, R. L., Landen, O. L. and Suter, L. J. Phys. Plasma, 11, 339 (2004).

[3] Meerson, B. Rev. Mod. Phys., 68, 215 (1996).

[4] Blondin, J. M., Fryxell, B. A. and Königl, A. ApJ, 360, 370 (1990).

[5] Hartigan, P., Reiter, M., Smith, N. and Bally, J. ApJ, 149, 101 (2015).

[6] Ichimaru, S. Rev. Mod. Phys., 54, 1017 (1982).

[7] Guillot, T. Science, 286, 72 (1999).

[8] Daligault, J. and Gupta, S. ApJ, 703, 994 (2009).

[9] Garcia Saiz, E. et al. Nature Phys., 4, 940 (2008).

[10] Knudson, M. D. and Desjarlais, M. P. and Dolan, D. H. Science, 322, 1822 (2008)

[11] Remington, B., A., Drake, R., P., and Ryutov, D., D., Rev. Mod. Phys., 78, 755-807 (2006).

[12] Rüter, H., and Redmer, R., PRL 112, 145007 (2014).

[13] White, T. G. et al. Phys. Rev. Lett., 111, 175002 (2013).

[14] Lindl, J. Phys. Plasmas, 2, 3933 (1995).

[15] Glenzer, S. H. and Redmer, R. Rev. Mod. Phys., 81, 1625 (2009).

[16] Gregori, G., Glenzer, S., H., Rozmus, W., Lee, R., W. and Landen, R., W. Phys. Rev. E, 67, 026412 (2003).
[17] Vorberger, J., Donko, Z., Tkachenko, I. M., and Gericke, D. O. Phys. Rev. Lett., 109, 225001 (2012).

[18] Mithen, J., P., Daligault, J., and Gregori, G. Phys. Rev. E, 83, 015401(R) (2011).

[19] Hansen, J-P. and McDonald, I. R. Theory of Simple Liquids, Academic Publications (2013)

[20] McDonald, I., R., Vieillefosse, P., and Hansen, J-P. PRL 39, 271 (1977)

[21] Mithen, J., P., Daligault, J., Crowley, B., J., B., and Gregori, G. Phys. Rev. E, 84, 046401 (2011).

[22] Cross, J. E., Reville, B and Gregori, G. ApJ, 795, 59 (2014).

[23] Schmidt, R., Crowley, B., J., B., Mithen, J. P., and Gregori, G. Phys. Rev. E, 85, 046408 (2012).

[24] Boon, J. P. and Yip, S. Molecular Hydrodynamics, Dover Publications (1991)

[25] Huba, J. D., Revised NRL Plasma Formulary. Office of Naval Research (2002).

[26] Ryutov, D. D., Drake, R. P., and Kane, J., ApJ, 518, 821-832 (1999).

[27] Tsakiris, G., D., and Eidmann, K. J. Quant. Spectrosc. Radiat. Transfer, 8, 353 (1987).

[28] MacFarlane, J., J., Golovkin, I.,E., and Woodruff, P., R. J. Quant. Spectrosc. Radiat. Transfer, 99, 381 (2006). 